\title{
CONTRACTS IN E-COMMERCE
}

\author{
Hans Weigand(h.weigand@kub.nl) \\ Lai Xu(l.xu@kub.nl) \\ Infolab \\ Tilburg University \\ The Netherlands
}

\begin{abstract}
Electronic Commerce involves coordination between different organizations using communication technology. The coordination can be achieved in the form of an electronic market or a virtual enterprise. In both cases, contracts play a central role. In the Electronic Market, contracts are agreements on a specific business transaction. In a Virtual Enterprise, the contract is a cooperation agreement for a longer period involving integrated business processes. A general framework of business communication is presented that is applied to a case study. We argue that an agent society model is most suitable for implementing contracts.
\end{abstract}

\section{Introduction}

The Electronic Market hypothesis ([Malone et al, 87]) predicted, on the basis of transaction cost theory, that the use of IT would cause a shift from hierarchies to markets. When communication costs go down, transaction costs are lower, allowing companies to concentrate on their core business and to buy external services on the market. Research in the '90s did not confirm this hypothesis ([Chirku \& Kauffman, 00]). One reason is that the costs of relating were insufficiently taken into account. In a long-term relationship between two companies, opportunistic behavior and fraud are less likely to happen. More mutual trust leads to less risk, and hence reduces the costs of risk management.

A Virtual Enterprise is a cooperation between independent companies that act together as a unit. The cooperation can be temporary, for the duration of one project or product stream, or longer. To act as a unit, the business processes must be integrated tightly. The integration can be achieved in various ways, but typically by means of interorganizational workflows (communication processes) on the one hand and shared databases on the other.

The original version of this chapter was revised: The copyright line was incorrect. This has been corrected. The Erratum to this chapter is available at DOI: 10.1007/978-0-387-35658-7_21 
In this paper, we first discuss the notion of "contract" as the focal point of interorganizational cooperation as well as in agent technology. Contracts can be used to provide semantics to the information exchanges in interorganizational processes. We describe a case of a virtual enterprise in the insurance domain. The case is analyzed within the contract framework. After that, in section 5, the implementation of this virtual enterprise in an agent society is discussed.

\section{Business contracts}

A contract is an agreement between two or more parties that is binding on those parties and that is based on mutual commitments. If there is only a one-sided commitment, most laws do not recognize this as a contract. Mutual commitment means that there is some exchange of goods and services. The most common case is that one party provides some service and the other party pays for it. For an agreement to be a contract, it is also important that there are enforcement mechanisms in place that ensure both detection and punishment of reneging ([Argyres \& Porter Liebesking, 99]).

Contracts have been at the core of traditional trade relations. Business transactions are usually divided into three consecutive phases: the contract preparation (or information phase), the contract negotiation (or agreement) and the contract fulfillment. In the most simple case, the fulfillment process is fixed as well as the price. In that case, there is not much to negotiate. The buyer selects a product, indicates that he wants to buy it, and the seller delivers it to him before, after, or at the same time as the payment. The process becomes more complex when the price is not fixed, as in most B2B commerce, when there are more issues to be agreed on than only the price, and when the delivery process is complex, as in international trade. In that case, the contract is more elaborate, including a specification in some form of the fulfillment process and an indication on how disputes are resolved.

It often pays off to the company to set up long-term relationships such as frame contracts, supply chain integration, and virtual enterprises. These contracts do not describe one particular business transaction, but provide the basis for many transactions during some period ([Atiyah, 89]). Such a contract can specify that party $A$ is authorized to inspect the inventory database of party $B$, and is obliged to replenish the inventory of B when a certain level is reached. In this way, the production and logistic planning in the supply chain can be optimized.

Related to contracts, several services can be identified that are traditionally fulfilled by social institutions but that can also be supported 
by electronic means. ([Milosevic et al, 95]) mentions the following: validation service, negotiation service (for instance, by using contract templates), monitoring service, enforcement service, arbitrating service (dispute handling), and repository service (providing legal information). In order for contracts to be processed automatically, a logical representation is needed, such as described for example by Daskalopulu ([Daskalopulu \& Sergot, 97]).

\subsection{Contracts and EDI}

In the past, companies used EDI and EDIFACT standards to communicate electronically. A drawback of EDI was formed by the high set-up costs. An EDIFACT agreement typically took a year or two for preparation. In the dynamic new economy, companies must be able to respond quickly to market changes. It should be possible to set up and dismantle new collaboration forms on a short term. The term "open-edi" has been introduced to describe the idea of EDI based on off-the-shelf agreements and protocols.

The challenge of the post-EDI generation is the ability to have an open set of messages rather than a predefined closed one. In the absence of a complete and comprehensive set of document formats, as EDIFACT did provide, several attempts are made to set up repositories of components that can be taken out and combined by business partners themselves. [Lee, 98] suggests the use of a central repository in which formal trade procedures can be stored. Users can download these trade procedures - formally represented as PetriNets -, adapt them if necessary, and then adopt them immediately for execution [Greunz et $a l, 00]$ describe an architecture for electronic contracts based on XML and logical representations that aims at supporting both the negotiation and the settlement phase. ebXML defines the broad notion of the TPA (Trading Partner Agreement) which specifies the capabilities of sending and receiving business documents ([ebXML, 00]). The TPA describes the configuration information and the interaction rules, which must be executable, can be automatically generated from the TPA in each party's system. The TPA is an XML document, the semantics are defined by a textual design document and are embodied in rules understood by the authoring tool, which aids in the creation of a valid TPA ([Sachs et $a l, 00])$. Although some of these approaches have been worked out in more detail than others, it is clear that a solution should include some semantic framework. Fixing the syntax is important, but not sufficient. Somehow, the intended effects must be described as well. 


\subsection{The role of contracts in formal business communication}

The formal language for business communication (FLBC) is a formal language based on speech-act theory that allows to specify EDI-like messages with a well-defined compositional semantics ([Kimbrough \& Moore, 97]). In its first version, FLBC has focused on the illocutionary semantics of individual messages. Recently, some work has been done to distinguish composites of messages "conversations" as well. In ( [Weigand \& Van de Heuvel, 98]; [Weigand \& Hasselbring]) messages are grouped into different aggregation levels of conversations, as displayed in figure 1.

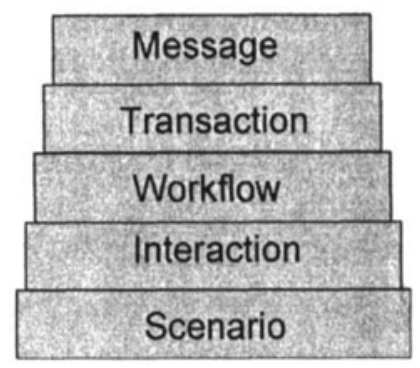

Figure 1. Conversational aggregation levels

At each level, various patterns can be defined. Messages (containing one or more speech acts) typically go in pairs, for instance a request followed by a response. The request/accept transaction is an example of a pattern at transaction level. A transaction is something that has an effect in the social world, such as the creation of an obligation. Transactions can be put together into workflows: a range of activities executed for the benefit of one principal (or initiator). Organizational coordination is usually based on reciprocity: the combination of two workflows that are symmetric in the agent/principal relationship. This aggregation level is called contracts or interactions. The contract specifies, among others, how the two workflows are synchronized. Finally, the scenario level describes the coherence between various contracts in the performance of a certain business process such as "sales".

The formal business communication framework aims at representing messages in e-commerce not on the data level, but on the semantic level. Part of the framework is currently applied in the European R\&D project MeMo that supports business negotiations for SMEs (see www.abnamro.com/ memo for more details about this project). 


\section{Multi-agent systems}

Contracts are at the core of economic interactions in human society. It is not surprising that the idea of "contract" reoccurs in the artificial societies known as multi-agent systems. One of the basic properties of agents is their relative autonomy. Hence an agent cannot be forced by another agent to perform a certain action, as in object worlds where any object can invoke any public method of any other object in the some domain. Just as business contracts allow for coordination in the economic world in the absence of hierarchies, agent societies need a coordination mechanism different from traditional top-down design. In the following, we discuss three different agent approaches using some notion of contracts.

\subsection{Contract Net Protocol}

In 1980, the Contract Net Protocol ([Smith, 80];[Smith \& Davis, 81]) for decentralized task allocation was one of the most important paradigms in distributed AI. In CNP, the network is assumed to consist of loosely coupled asynchronous agents. The agents are interconnected so that each agent can communicate with every other agent by sending messages. The Contract Net Protocol (CNP) is a simple negotiation protocol. In the role of contractor, an agent can decompose a task into subtasks, send request for bids on each specific subtask to all the other agents, select the most appropriate bid and allocate the task to that subcontractor. The protocol assumes that all agents are eager to contribute, and the most appropriate bid is the bid of the agent with the best capability and availability.

A more sophisticated version of the CNP was developed by Sandholm in the TRACONET model ([Sandholm \& Lesser, 95]). In this model, agents are supposed to be self-interested. This means that contractors have to pay a price for the service performed. Contractors try to minimize the costs by selecting the bidder with the lowest price (all things being equal). Potential subcontractors try to maximize its benefit. If they read an announcement of a contractor that offers a price lower than their minimum price, they will discard it. It is also possible to respond by a counter offer.

\subsection{Cooperative Information Agent}

A Cooperative Information Agent (CIA) model was described by Verharen in his Ph.D. thesis ([Verharen, 97]) and is based on the Language/Action Perspective. The CIA model assumes that the agent be- 
havior is not predefined but based on commitments to other agents. These commitments are specified in contracts. The agent has a number of tasks that it can be perform (in principle, an extensible set). Which tasks are executed, is determined by the contracts that the agent has set up with other agents. The model assumes that contracts are set up by the human designers, in the same way as an Information System is designed, with the difference that the agent contract is based on an agreement between at least two parties (for example, two companies). However, the model does also allow agents to send unauthorized requests to other agents; the receiving agent can ignore such a request, but it can also respond by proposing a contract (with mutual commitments). If the first agent accepts the proposal, there is a contract, and they can start authorized interactions.

The semantics of contracts can be described by means of illocutionary deontic logic, the logic of obligations, authorizations and speech acts. For more details, we refer to ([Weigand \& Dignum, 97]).

\subsection{Contractual Agent Societies}

Contractual Agent Societies (CAS) ([Dellarocas \& Klein, 99]; [Dellarocas, 00]) are open systems where independently developed agents configure themselves automatically. They coordinate their behavior through a set of dynamically negotiated contracts, which define the shared context of agent interactions, and a system of social control, which is responsible for avoiding, or detecting and resolving exceptions (including malicious behavior and non-compliance). Crucial to the CAS model is the distinction between mutually trusted agents and mutually untrusted agents. A market place is a set of mutually trusted agents; when an untrusted agent wants to join the market place, it applies at a socialization service that not only plugs in the agent technically, but also makes him agree on a social contract. A social contract is a commitment of an agent to participate in a society (or market place) and obey its norms. Within the society or market place, agents can set up private contracts on a bilateral basis, for example, by means of CNP or TRACONET. The society commits itself to enforce the agent's private contracts. To this end, two special agents are defined: a notary agent, responsible for storing contracts and resolving potential disputes, and a reputation agent, responsible for keeping records of all contracts formed by members of the market place - including breaches. Malbehaving agents can be banned from a society, if this is specified in the social contract. The society also contains a matchmaker agent that helps registered agents to locate other members. 


\subsection{Evaluation}

The CNP protocol is useful in the context of distributed problem solving, but too naive for situations where the agents are involved in real business. TRACONET is more realistic, but does not cope with opportunistic behavior. TRACONET can be applied in business situations, such as Electronic Markets, when there is some umbrella contract or a market owner with enforcing power. However, legal context is outside of the scope of TRACONET. CAS brings the legal context into the model by means of the social contract mechanism. To make it works in practice, it is still necessary to embed the agent society in the "real world", in order to give the social contract a legal basis. CAS is not very specific about the nature of the private contracts. The private contracts are supposed to be instances of one of the protocols in the social norms knowledge base. Contracts such as used in the CIA model can be used for both single transactions and long-term agreements.

In the following, we will apply the formal business communication model to an insurance domain case, focusing on the modelling of contracts. Subsequently, we will show what how these contracts are implemented in an agent society infrastructure such as CAS.

\section{Case: Insurance Domain}

This case outlines the manner in which a private motor damage claim is handled by AGFIL ([Browne \& Kellett, 99]), it describes the interaction between motor damage team of AGFIL and various third parties, each participating party shares the common objective to provide a service level which facilitates efficient claim settlement, the process is shown in Figure 2.

\subsection{Case description}

The participating parties involved in the case are Europ Assist, Lee Consulting Services, Garages and Assessors. Europ Assist offers a 24hour emergency call answering service to AGFIL's motor and household policyholders. Lee C.S. coordinates and manages the operation of the emergency service on a day to day level on behalf of AGFL. The Garages used in the case are referred to as "Approved Repairers" and offer a discount on repair costs to AGFIL. The panel of assessors is dictated by AGFIL. The Assessors conduct the physical inspections of damaged vehicles and agree repair figure with the garages.

The policyholder phones Europ Assist using a free-phone number to notify a new claim. Europ Assist will register the information on an inci- 


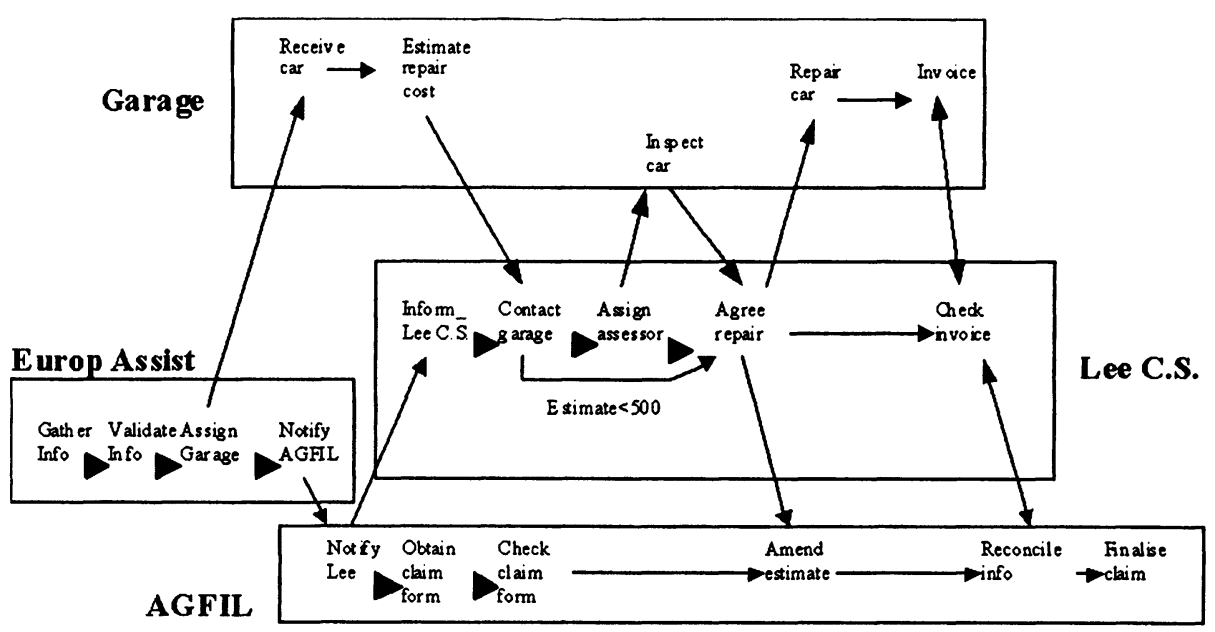

Figure 2. Informal diagram of AGFIL process

dent form and give some suggestions about garage to policyholder. The incident form is then sent to AGFIL and Lee C.S. replicate the AGFIL database to receive notification details. The AGFIL claim handler will check to confirm cover. In the event of the claim being invalid, Lee C.S. will be contacted and the process will be stopped. Lee C.S. will agree repair figures if an adjuster/assessor is not required or will otherwise either appoint an assessor or conduct the assessment themselves.

When repairs are completed the garage will issue an invoice to the Adjuster/Assessor who will check the invoice against the original estimate. Lee C.S. sends a monthly borderaux to AGFIL listing all repairs for that month.

The negotiation will take place if the revised figure is higher. AGFIL will contact Lee's who will investigate the situation through discussions with the garage and/or assessor. If the motor accident has resulted in an injury to a passenger and/or a third part, the handling of the entire claim will be transferred to personal injury team of AGFIL.

\subsection{Case analysis}

An analysis of the case starts by first considering the contract between AGFIL and the policyholder. In this contract, the services of AGFIL are described, corresponding to authorizations of the policyholder. The other contracts are outsourcing contracts that are instrumental to AGFIL for fulfilling its contract with the policyholder. 
The formal representations are a simplified form of Deontic Dynamic Logic (see [Weigand \& Dignum, 97] for more details). Most important is the modal operator $O B L(\alpha)$ for obligation that takes an action as argument, and the modal dynamic operator $[\alpha] \phi$ that says that after action $\alpha$ is performed, $\phi$ holds.

$A: O B L($ let_repair $(x)) \Longleftarrow(P E R F:$ damage $(x, t)) \wedge$ car $(x) \wedge i n_{-}$term $\left.(t)\right)$ $P: O B L(\operatorname{pay}(d, t)) \Longleftarrow(\operatorname{premium}(P)=d \wedge t=" D e c, 1 ")$

The obligation of AGFIL to let repair the car when it is damaged induces a control loop with an actagenic transaction (REQUEST / COMMIT) before and a factagenic transaction (ASSERT / ACCEPT) after the execution of the action. These transactions must be implemented using some communication system. In this case, the actagenic transaction is implemented using a toll-free telephone. The factagenic transaction is not described in the case, but presumably handled by a paper letter. Similarly, the obligation of $\mathrm{P}$ to pay the premium leads to two transactions, as well as a money transfer action, but these are not in the scope of the case.

The repair is an explicitly outsourced action. The agent is the garage. This leads to a second contract between AGFIL and the garage:

$G: O B L($ repair $(x))$

$A: O B L(p a y(e)) \Longleftarrow \operatorname{cost}(x)=e$

In contrast to the first contract, this contract is specific to one situation. In such a case, the preparation of the contract coincides with the enactment of the contract. Only in the case that one of the parties is negligent in fulfilling its obligation, the other party needs to make a claim.

The preparation of the contract is realized in a negotiation transaction that results in the two obligations. Negotiation transactions can be more or less elaborate. In the simple case, the messages are ORDER and COMMIT(ORDER), possibly backed up with a discussion layer consisting of proposals and counter-proposals. In a more elaborate case, there is not one transaction, but a workflow of two transactions: first a transaction in which a quote is asked for, and then a transaction in which the contract is made. The quote transaction consists of a REQUEST(QUOTE), a QUOTE and a ACCEPT(QUOTE). In the AGFIL case, the costs of the repair must be estimated, so the more elaborate negotiation scheme is more appropriate. After the execution of the repair action, the garage reports to AGFIL using a factagenic transaction. 
The specific contract between Garage and AGFIL is not negotiated from scratch. The case description explains that there is a list of approved garages that also offer discount to AGFIL. So the specific contract is made within the context of an umbrella contract, a generic contract that allows AGFIL to request repair against a discounted price.

$$
\begin{aligned}
& A: \operatorname{auth}(R E Q U E S T(A, G, \operatorname{repair}(G, x, p))) \Longleftarrow \\
&(\operatorname{Garage}(G) \wedge \operatorname{car}(x) \wedge \text { discount_price }(p))
\end{aligned}
$$

An authorization is a deontic state that gives $A$ the right (which is more than a permission) to request the repair of a car against a discount price. This authorization can be used in the above-mentioned negotiation between $A$ and $G$ when $A$ makes a claim on discount.

It would be possible to stop here, but AGFIL has decided to outsource more actions. The communication with the policy holder (which for AGFIL consists of awaiting requests, and then accepting) is delegated to a call center, Europe Assist. This leads to a new contract between AGFIL and Europe Assist:

\section{$A: O B L(p a y(f))$ \\ $E: O B L(G E T($ request $(r)) \wedge O B L(A C C E P T($ request $(r, y))) \Longleftarrow$ $(P E R F: G E T($ request $(r, y)) \wedge \operatorname{valid}(r, y))$}

The new contract does not induce a new actagenic transaction, since the claim handling is initiated by the policy holder y. However, it does lead to one or more new factagenic transactions in which Europe Assist reports about the fulfilment of its obligations: the claims that it has received and the acceptances it has made. The incident form could be the medium by means of which Europe Assist reports to AGFIL, but a better interpretation (given the data in the case description) is to view the incident form as a forwarding of the claim to AGFIL. This forwarding is necessary because the claim is still a claim of the policy holder to AGFIL; the call center is only intermediary. That is why we have split up the factagenic transaction in a REQUEST, ACCEPT and COMMIT part. The ACCEPT is only the uptake (Austin - "I have your message"), the COMMIT leads to a new deontic state. Normally, ACCEPT is part of the COMMIT, but in this complex outsourcing situation, the two have been separated. In passing, we note that AGFIL must be aware of the confusion that could arise because of this separation, and must communicate clearly to the policy holder at which point the claim is really committed.

AGFIL has also outsourced its communication with the Garage. Both the actagenic transaction (the request to repair the car, including a 
commitment to a certain price) and the factagenic transaction (the conclusion that the car has been repaired), as well as the handling of the counter-actagenic transaction (the request to pay from the Garage to AGFIL) are performed by Lee C.S. on behalf of AGFIL. All these transactions consist of at least a REQUEST and COMMIT, but they can include negotiations and information exchanges as well.

Finally, Lee C.S. itself has decided to outsource one of its actions to an Assessor in the case that it cannot estimate the damage itself. The outsourced action is estimate_damage is instrumental to its actagenic conversation - it has to know the damage before it can commit to a certain price, since the price that the Garage asks for is based on its estimation of the damage. It is not sufficient for the two parties to agree on the fact that the car is damaged, they must also agree on the amount of damage. In fact, the estimation of the damage is not only instrumental to the price negotiation, but already to the REQUEST itself. Normally, Lee C.S. will not just request the garage to repair the car, but will request to repair the car on this and that point. For that reason, the actagenic transaction is expanded to include an informative transaction in which both parties agree on the situation definition (Verständigung, in Habermas' terms).

Interestingly, the communication between AGFIL and Lee C.S. is by means of a shared database rather than a message exchange. Lee C.S. has the contractual obligation to process the claim, more specifically, to contact the garage, as soon as there is a claim. So the obligation does not need to be established anymore. The only thing that is required is that Lee C.S. receives the necessary information. The contractual obligation of Lee C.S. should be specified clearly in the contract.

The analysis leads to a scenario in which all the stakeholders are represented and the kind of contracts between them. We have shown in the analysis that not just any message sequence is a meaningful scenario. The meaningful scenario's can be generated by a kind of generative grammar, that starts with a simple two-sided contract, and is transformed step by step by expanding certain steps and/or introducing an agency relationship. The advantage of such a grammar is that it can be of great help in the design process of an e- business scenario; it allows the designer to derive meaningful scenario's only, and offers a high abstraction level. Compare this to the situation where the communication is described using message sequence charts or document flow charts. 


\section{Implementation of the Insurance Domain Agent Infrastructure}

In this insurance domain example, the application intrinsically involves cross-organizational workflows, and thus some forms of workflow technology have been used for certain parts system. Agent technology and workflow technology should cooperate effectively; the agent technology can be used to make workflow more intelligent. Today, the activities are initiated and executed primarily by humans. In the future, with the increasing automation, they can be supported by software agents. We design the insurance domain as an agent-based e-marketplace that is very similar to conventional social institutions. In the agent-based insurance domain, independently developed agents configure themselves automatically and coordinate their behavior through a set of dynamically negotiated social contracts, which define the shared context of agent interactions, and a system of social control. Figure 3 depicts the insurance marketplace infrastructure.

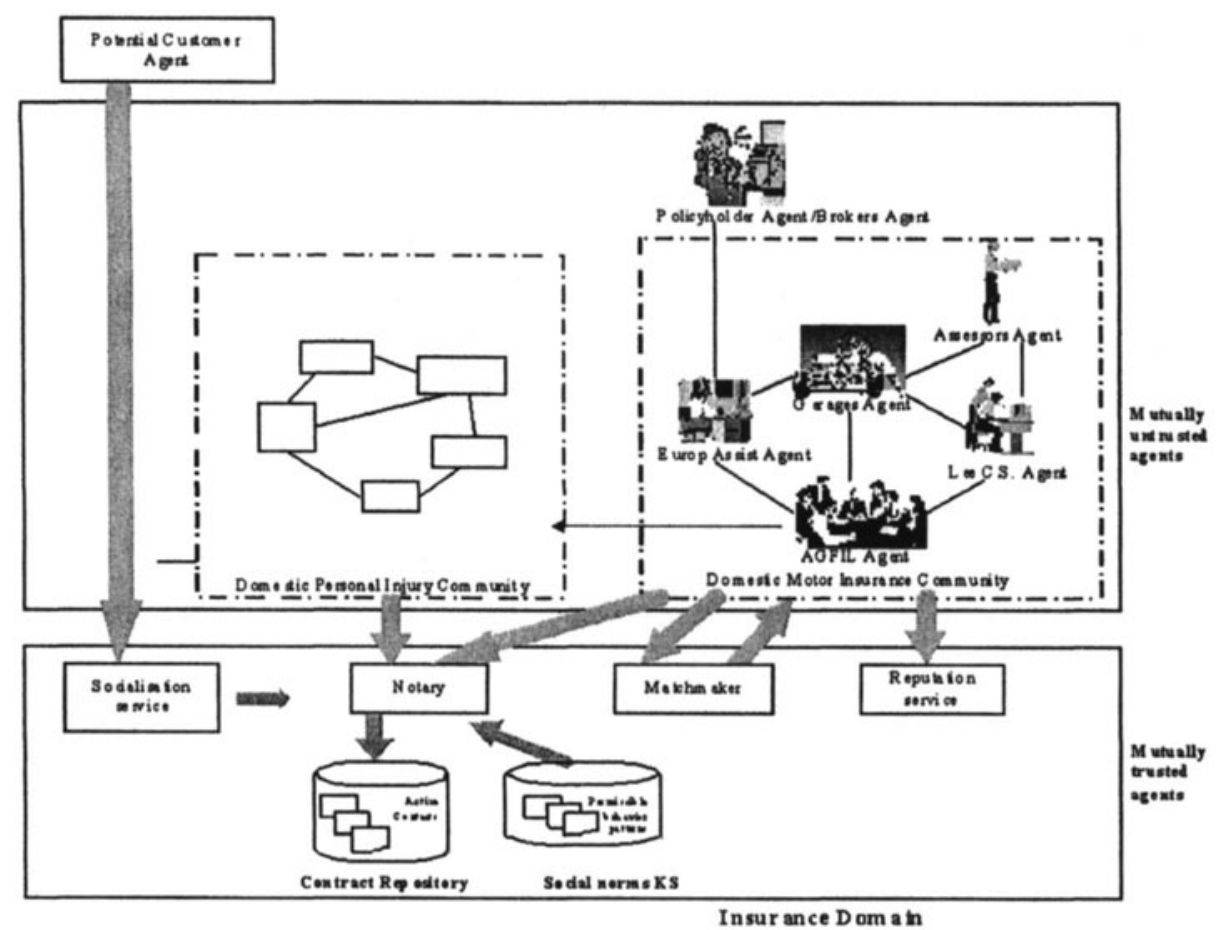

Figure 3. Agent-based insurance e-marketplace 
The infrastructure of the agent-based insurance domain includes mutually trusted and untrusted agents. Mutually trusted agents include the socialization agent, the matchmaker the notary agent and the reputation agent to enable the agent-based insurance marketplace to control all members and to keep the marketplace security.

In order for (possible heterogeneous and untrusted) agents to join the marketplace, they first have to negotiate social contracts with the socialization agent. The process of socialization is an enhanced version of the registration process of other agent environments.

We can assume that the AGFIL agent has set up the marketplace and has asked a Europ Assist agent and a Lee C.S. agent to originate a virtual motor insurance community. We assume that this group is kept stable. However, Garage agents and Assessor agents may enter the marketplace and leave at different points in time. Suppose that the Lee C.S. agent is looking for an Assessor or Garage. To locate an agent in the agent-based marketplace, members must send a RFB (Request for Bids) message to the matchmaker, describing the requested service (e.g. the assessment). The matchmaker then broadcasts the request to all potentially eligible members. Interested members may then contact the sender directly by sending it a BID message. After they locate one another, they use exactly the same language they used to interact with the socialization service agent in order to negotiate a new private contract, which will define their partnership.

Once an acceptable bid has been received, the two parties can start communicating directly, or else negotiate and form a contract through the notary service. The benefit of forming contracts is that the marketplace then offers a number of "legal" guarantees. If a contract is unilaterally cancelled by one of the parties, the notary service informs the reputation agent. Also, if a contract is breached the notary informs both the reputation agent and the matchmaker. Since AGFIL is market owner in this case, it is up to AGFIL to take remedial action, for example, to ban a certain agent from the marketplace.

\section{Conclusion}

Contracts are used in electronic markets, but also in virtual organizations. Analyzing e-commerce cases, such as the insurance case that was adopted in this paper, from a contract perspective, means that the focus is not on individual messages, but on business relationships. Within each relationship, there is a reciprocity. Within an agency relationship, there is a control loop. From a contract perspective, a certain e-business case, however complex, is the result of outsourcing and decomposition 
processes that can be represented formally by means of a generative grammar. Any semantics of business communication should account for contracts, but also current design methods should be extended. A message-oriented design method such as UML cannot account for the coherence in business communication.

When contracts are taken as pivotal elements in e-commerce, the implementation framework must be in line with this perspective. In our opinion, the Agent Society model is very suitable. The business contracts can be mapped onto private contracts between agents. The social contracts provide the necessary embedding of the agents in context. For reasons of space, we have left out the specifications of the social and private contracts. We are currently working on the implementation of a prototype Agent Society.

\section{References}

Argyres, N.S., Porter Liebesking, J., 1999. Contractual Commitments, Bargaining Power and Governance Inseparability: Incorporating History into Transaction Cost Theory. Academy of Management Review, Vol. 24, No.1, pp.49-63.

Atiyah, P.S., 1989. An introduction to the Law of Contract. Clarendon Press, Oxford. Browne. S. and M. Kellett, 1999. Insurance Scenario, CrossFlow Report D1.a.

Chirku, A. \& R. Kauffman, 2000. Reintermediation Strategies in Business-to-Business Electronic Commerce. Int. Journal of Electronic Commerce, 4(4), pp.7-42.

Daskalopulu, A. \& M.J. Sergot, 1997. The representation of legal contracts. AI and Society, 11 (1\&2), pp.6-17.

C. Dellarocas \& M. Klein, 1999. Civil Agent Societies: Tools for inventing open agentmediated electronic marketplaces. Proc of the Workshop in Agent-Mediated Electronic Commerce (co-located with IJCAI'99), Stockholm, Sweden, July 1999.

C. Dellarocas, 2000. Contractual Agent Societies: Negotiated shared context and social control in open multi-agent systems. Workshop on Norms and Institutions in Multi-Agent Systems, Agents-2000, Barcelona, June 2000.

ebXML, 2000. ebXML Technical Architecture Team, ebXML Technical Architecture Specification, October, 2000.

FIPA (Foundation for Intelligent Physical Agents). See:

http://www.fipa.org.

Greunz, M. \& K. Stanoevska-Slabeva \& B. Schopp, 2000. Electronic Contracting with XML containers. In: Informations-systeme für e-commerce. EMISA-2000, Nov, 810, Linz, pp.11-27.

Kimbrough, S. \& S. Moore, 1997. On automated message processing in electronic commerce and work support systems: speech act theory, and expressive felicity. ACM Trans on Information Systems, 15 (4), pp.321-3367.

Lee, R., 1998. Towards open electronic contracting. Int. Journal of Electronic Markets, pp3-8.

Malone, T.W. \& Yates, J. \& Benjamin, R.I., 1987. Electronic markets and electronic hierarchies. Comm. of the ACM 30,6, pp.484-497. 
Milosevic, Z \& Berry, A \& Bond, A \& Raymond, K., 1995. An architecture for supporting business contracts in open distributed systems. Int. Workshop on Services in Distributed and Networked Environments, Whistler, Canada.

Sachs M. \& Dan A, Nguyen T. \& Kearney R. \& Shaikh H. \& Dias D., 2000. Executable Trading-Partner Agreements in Electronic Commerce. IBM Corporation.

Sandholm T. \& Lesser V., 1997(1995). Issues in Automated Negotiation and Electronic Commerce: Extending the Contract Net Framework. In: M. Huhns, M. Singh (eds), Readings in Agents, Morgan Kaufmann.

Smith R. G., 1980. The Contract Net Protocol: High-Level Communication and Control in a Distributed Problem Solver, IEEE Transactions on Computers, vol. C-29, No. 12,1980

Smith R.G. \& Davis R., 1981. Frameworks for Cooperation in Distributed Problem Solving, IEEE Transactions on Systems, Man, and Cybernetics Vol. SMC-11, No.1.

Verharen, E., 1997. A Language/Action Perspective on Cooperative Information Agents. Ph.D. Thesis, Tilburg University..

Weigand, H. \& F. Dignum, 1997. Integrated semantics for information and communication systems. In: R. Meersman (Ed), Proc. WG 2.5 Database application semantics, Chapman \& Hall.

Weigand H. \& W.J. van de Heuvel, 1998: Meta-patterns for Electronic Commerce based on FLBC. Proc. HICSS'98, IEEE Press.

Weigand, H. \& Hasselbring, W., 2000 An extensible language for business communication. Proc. COOPIS '2000, LNCS, Springer-Verlag. 